Journal of Computer Science 6 (7): 693-699, 2010

ISSN 1549-3636

(C) 2010 Science Publications

\title{
Face Biometrics Based on Principal Component Analysis and Linear Discriminant Analysis
}

\author{
Lih-Heng Chan, Sh-Hussain Salleh and Chee-Ming Ting \\ Center for Biomedical Engineering, Faculty of Biomedical Engineering and Health Science, \\ University Technology Malaysia, 81310 UTM Skudai, Johor DT, Malaysia
}

\begin{abstract}
Problem statement: In facial biometrics, face features are used as the required human traits for automatic recognition. Feature extracted from face images are significant for face biometrics system performance. Approach: In this thesis, a framework of facial biometric was designed based on two subspace methods i.e., Principal Component Analysis (PCA) and Linear Discriminant Analysis (LDA). First, PCA is used for dimension reduction, where original face images are projected into lower-dimensional face representations. Second, LDA was proposed to provide a solution of better discriminant. Both PCA and LDA features were presented to Euclidean distance measurement which is conveniently used as a benchmark. The algorithms were evaluated in face identification and verification using a standard face database-AT and $\mathrm{T}$ and a locally collected database-CBE. Each database consists of 400 images and 320 images respectively. Results: LDA-based methods outperform PCA for both face identification and verification. For face identification, PCA achieves accuracy of $91.9 \%$ (AT and T) and 76.7\% (CBE) while LDA 94.2\% (AT and T) and 83.1\% (CBE). For face verification, PCA achieves Equal Error Rate (EER) of $1.15 \%$ (AT and T), $7.3 \%$ (CBE) while LDA $0.78 \%$ (AT and T) and $5.81 \%(\mathrm{CBE})$. Conclusion/Recommendations: This study had proved that, when given sufficient training samples, LDA is able to provide better discriminant ability in feature extraction for face biometrics.
\end{abstract}

Key words: Face biometrics, identification, verification, principal component analysis, linear discriminant analysis, equal error rate

\section{INTRODUCTION}

Biometric is the technology of measuring intrinsic physical and behavior traits of human for authentication purpose. Emerging of this technology is motivated by traditional methods limitations in identity verification. Traditional methods can be categorized as possessionbased and knowledge-based methods. In possessionbased method, items such as cards, badges, or keys are utilized. This method requires low cost. However, these items can be shared, duplicated and easily lost or stolen. In knowledge-based method, there are also drawbacks for those who used password or PIN. Some passwords are easily guessed. Besides, they can be shared or forgotten.

Facial biometric depends on the recognition engine which base on face features as the required human traits. Compared with other very reliable methods such as fingerprint and retinal scans, human face stand an advantage in terms of collectability, where low level of user's cooperation is required (easy to obtain and quantified with a sensor).

Growth of facial biometrics is spurred by evolution of computer vision research. This evolution has been triggered by the mushrooming commercial and lawenforcement applications and availability of feasible technologies (Zhao et al., 2003). Although the machine recognition of faces has reached a certain level of maturity, yet technological challenges still remain in many aspects, such as illumination changes, pose variation, aging. As a result, researches are still on going to further advance current state of the art which involves various aspects. Even psychology or cognitive study has become part of the research to provide inspiration on improving face recognition.

Identification and verification: There are two modes in which biometric systems can perform: identification and verification.

Corresponding Author: Lih-Heng Chan, Center for Biomedical Engineering,

Faculty of Biomedical Engineering and Health Science, University Technology Malaysia,

81310 UTM Skudai, Johor DT, Malaysia Tel: +60125311802 Fax: +6075535430 


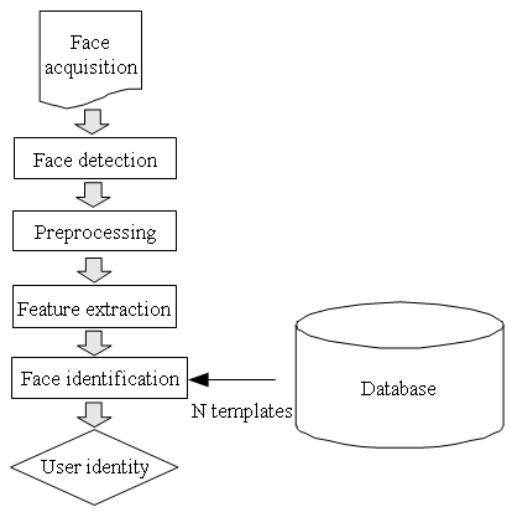

Fig. 1: Block diagram of face identification

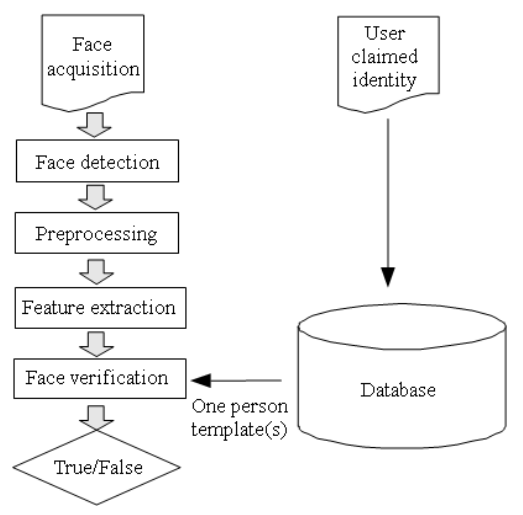

Fig. 2: Block diagram of face verification

In face identification, once a user has his/her face sample presented to biometric system, the sample is compared with every biometric reference stored in database. Face identification complete procedures are shown in Fig. 1. Face acquisition module is the entry point, where face images are captured by camera. Face detection locates and segments certain face region from cluttered scenes. Alternately, several preprocessing techniques can be applied on face images for enhancement, such as histogram equalization, filtering, background removal, illumination normalization, size normalization. Afterwards, relevant features are extracted from face images and presented to classification based on identification concept. Face identification system has to determine the identity of the input face image based on comparisons among all templates stored in database. It is an one to many (1: N) application. This application is typically used for surveillances and law enforcement investigations.

Second, in face verification, user's face template is compared with specific face biometric references in the database corresponding to the user's identity. While giving biometric sample, the user has to claim identity by entering name, or presenting ID cards, token as shown in Fig. 2. With this claimed identity, biometric system compares the biometric sample with associated biometric references. Finally the system yields verification results i.e., true or false. Verification is a one to one (1:1) concept which is commonly applied in access control applications.

PCA and LDA: Kirby and Sirovich (1990) first introduce adoption of Karhunen-Loeve into face processing. Karhunen-Loeve also goes with the name of PCA or Hotelling Transform. They show that, face images can be represented by eigenpictures which span a small dimensionality. In other words, any particular face can be economically represented in terms of a best coordinate system. This demonstration inspires and leads to the first successful automatic face recognition system by Turk and Pentland (1991), which propose the so-called eigenface method. Eigenface approach is derived by applying PCA on covariance matrix of a face dataset to find vectors that best account for the distribution of face images. These vectors are eigenvectors of covariance matrix corresponding to original face images. The created PCA projection space contains the significant variations among those face images. Any face image can be projected on the PCA space, giving a low dimensional representation of face.

However, PCA method suffers the disadvantage in terms of discriminant ability. Its performance deteriorates especially in the present of varying lighting condition and facial expression. At training stage, eigenvectors are obtained based on maximization of the total scatter matrix. In other words, significant features that cause large variations between training images are emphasized by PCA. Thus, some unwanted changes are retained, such as light variation and face expression.

Thus, LDA have been proposed to improve discriminant ability of PCA. The combination of PCA and LDA has been proven of achieving relevant good results (Belhumeur et al., 1997; Zhao et al., 1998). When given sufficient sample size, LDA can outperform PCA (Martinez and Kak, 2001; Ruiz-del Solar and Navarrete, 2005).

\section{MATERIALS AND METHODS}

Method and development: Turk and Pentland (1991) proposed the following steps in developing eigenface method for face recognition. Steps for training are as:

- Acquire an initial set of face images which are appointed as training samples

- Calculate the eigenfaces from the training set. Keep only $\mathbf{M}^{\prime}$ eigenfaces which correspond to 
largest eigenvalues. These eigenfaces span the face space. If there are new face images to be included in training sets, this face space has to be recalculated (update)

- Calculate the weights of each individual face images which have contributed in training set. This is done by projection the images onto the face space

After initializing the system, the following steps are carried out for recognition of new face images:

- When a new face image is presented, project it on face space to obtain its set of weights

- Based on the weighs, determine whether the image represent a face. This is done by checking whether the image is sufficiently close to the face space

- If it is a face, classify the weight pattern as either a known person or unknown

- (Optional) update eigenfaces

- (Optional) If the same unknown faces are encountered several times, the system may calculate its weights pattern and incorporate into known faces

In this study, procedures 2-5 are modified regarding the objectives. In identification, the new input image proceeds directly to identity classification. Instead of finding out known or unknown person, our algorithms classify and determine the identity of the input image. The flowchart of the algorithm is depicted in Fig. 3.

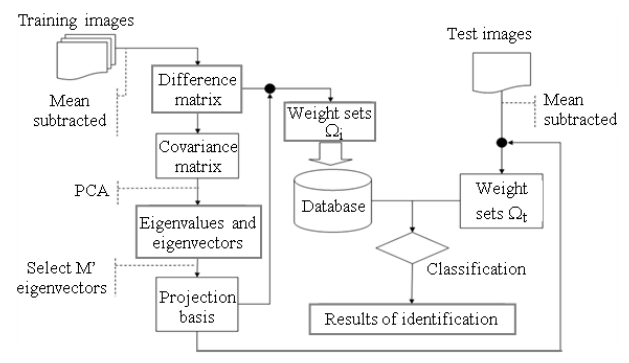

Fig. 3: Block diagram of PCA method

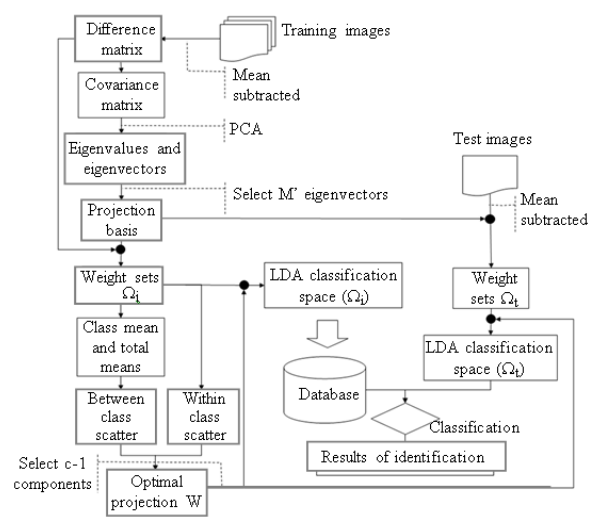

Fig. 4: Block diagram of LDA method
PCA is a standard technique to represent original data with lower dimensionality. On the other hand, LDA finds an optimal linear discriminant function to map the input into the classification space. Therefore, this LDA method involves double projections i.e., PCA projection and LDA projection. Fig. 4 shows a complete flowchart of LDA. Class information in weight sets $\Omega_{\mathrm{i}}$ are exploited to calculate between-class scatter matrix and within-class scatter matrix. Both scatter matrixes are used to formulate criteria for class separation. To use class information, LDA must have at least two face images per person for training.

Within-class scatter matrix is a sum of covariance of each of class; while between-class scatter matrix is a covariance of dataset whose members are mean vectors of each class. The optimization criterion in LDA is the ratio of between-class scatter matrix to that of withinclass scatter matrix. Weight vectors of PCA space can be projected into LDA classification space $\mathrm{g}\left(\Omega_{\mathrm{i}}\right)$ based on optimal projection. Face images represented in $\mathrm{g}\left(\Omega_{\mathrm{i}}\right)$ are expected to be more discriminative than original images in PCA space.

Database: The $\mathrm{AT}$ and $\mathrm{T}$ (AT and $\mathrm{T}$ Laboratories Cambridge, 2002) which is formerly known as ORL face database. It contains 10 different images of 40 distinct subjects, giving a total of 400 images. They vary in terms of lighting, facial expressions including open/closed eyes, facial details such as glass/without glasses and different time of snapping pictures. All the images were taken against a dark homogeneous background with the subjects in an upright, frontal position (with tolerance for some side movement). Figure 5 shows a sample subject of AT and T database.

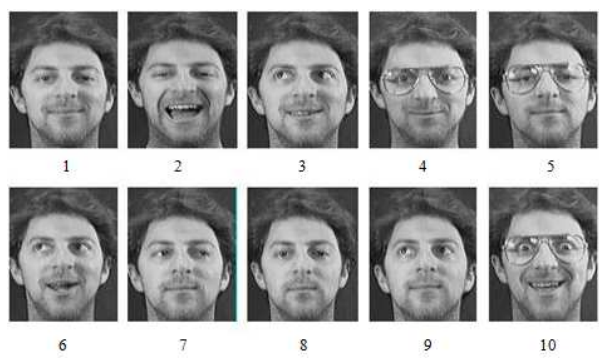

Fig. 5: Sample of AT and T database

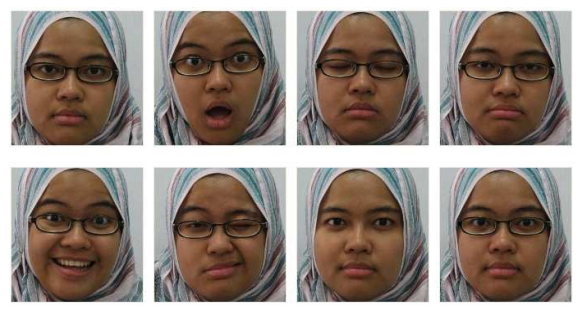

Fig. 6: Sample of CBE database 
The second database used for face biometrics evaluation is Centre for Biomedical Engineering (CBE) face database which was collected in September 2007 till October 2007. It is a local face database contributed by University Technology Malaysia (UTM) voluntary undergraduates who were around 20-22 years-old. These images were captured against a white background with the subject in an upright, frontal position. There are 40 subjects and each subject has 8 images, yielding a total number of 320 images. Images of a single subject vary mostly in terms of face expression such as normal, surprise, sleepy, sad, happy and wink. Besides, some images vary in terms of head orientation around $10-30^{\circ}$ and also face details such as glasses/no glasses. Figure 6 shows a sample subject of CBE database.

Identification parameter selection: First, PCA method performance under variation of principal components is studied using AT and T dataset. Figure 7 shows PCA performances with different amount of principal component selected. PCA recognition rate is found improving drastically when the number of principal components, PC is increased from 2-15. Its performance levels off at around $89 \%$ starting from $\mathrm{PC}=35$.

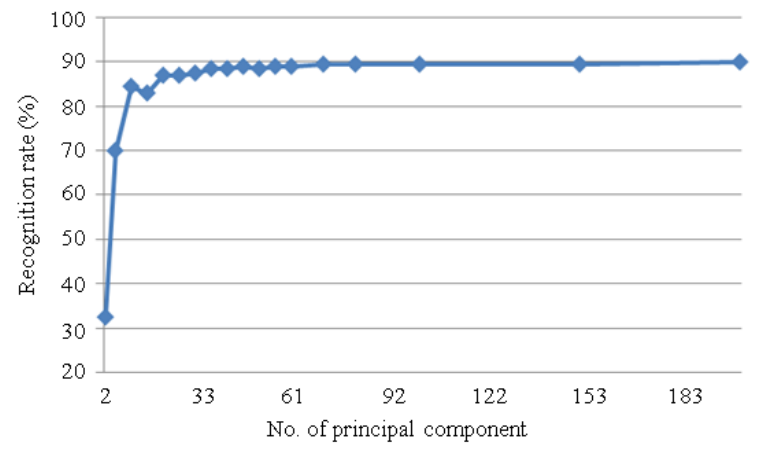

Fig. 7: Recognition rate of PCA under varying PC for $\mathrm{AT}$ and $\mathrm{T}$ face database

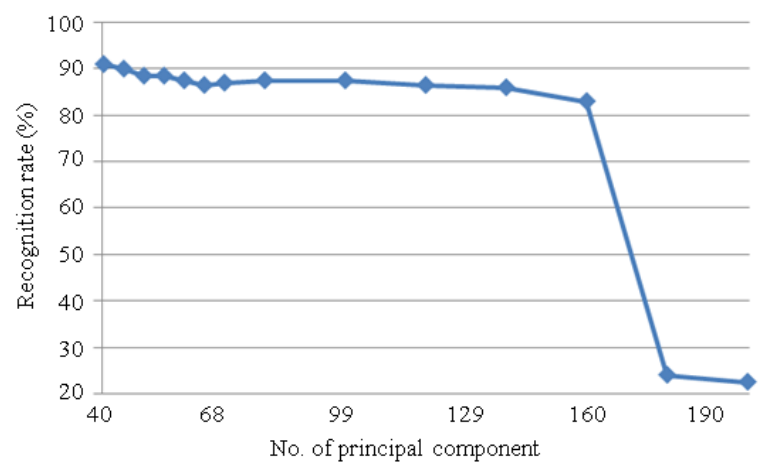

Fig. 8: Recognition rate of LDA under varying PC for $\mathrm{AT}$ and $\mathrm{T}$ face database
Second, LDA subspace method performance is investigated using various PC. Figure 8 shows the experimental results of LDA with PC lie in the range of 40-200. Highest recognition rate of LDA is achieved at $\mathrm{PC}=40$. However, LDA performance deteriorates significantly when more than 150 PC are used. Instead, $\mathrm{PC}=40$ is the most optimum choice for creating LDA subspace. Based on the study, $\mathrm{PC}=40$ are selected for both PCA and LDA methods.

Effect of varying $\mathrm{PC}$ is also studied in CBE Face Database using PCA and LDA methods. Figure 9 and 10 show evaluation performances of PCA and LDA under varying $\mathrm{PC}$ which lie in the ranges of 2-200 and 40-200. It is found that the performances patterns are similar to PCA and LDA using AT and T. Based on the study, $\mathrm{PC}=60$ is selected for PCA while $\mathrm{PC}=45$ is chosen for LDA.

Verification experimental protocols: Performances of algorithms in face verification were evaluated using two databases i.e., AT and T and CBE face database. Experiments were run based on two protocols to double validate algorithms performances. In this thesis, the protocols are abbreviated as PROTO1 and PROTO2.

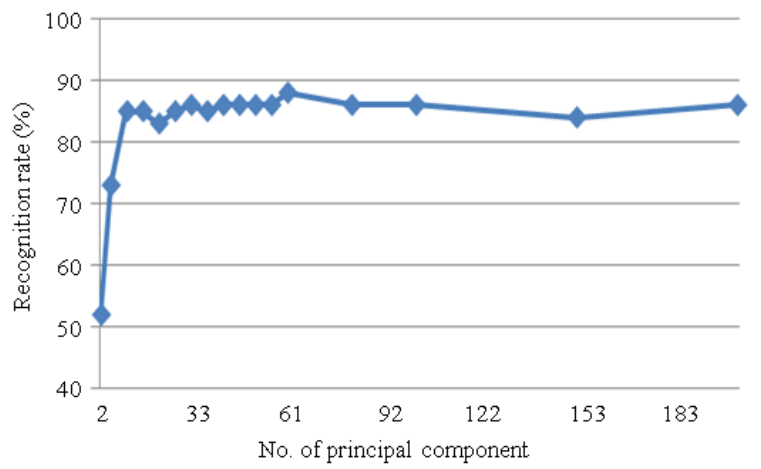

Fig. 9: Recognition rate of PCA under varying PC for CBE face database

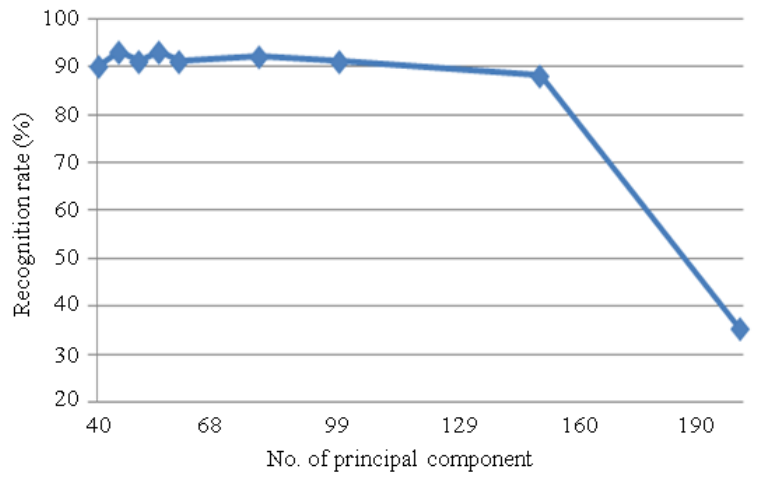

Fig. 10: Recognition rate of LDA under varying PC for CBE face database 
In PROTO1, experiments were implemented by adopting the combination of the left-one-out and the rotation estimates i.e., a variant of the jack-knife (Kotropoulos et al., 2000; Goudelis et al., 2007). This protocol has been used in multimodal authentication. Let the term shot denote the collection of one frontal face image per person. AT and $\mathrm{T}$ is considered for this protocol explanation. Thus, there are 10 shots per subject. Forty subjects are labeled as M1-M40.

First, regarding Fig. 11, 1 subject, i.e., M40 is appointed as imposter while the rest of 39 subjects are labeled as clients; 1 shot of every subject is selected as test set while the rest 9 shots of clients build the training set. For this combination, 39 authentic tests and 39 imposter tests can be performed.

Second in series rotation, 10th serial of shot are rotated to play the role of training set; while 9th serial is rotated to test set in different combination. Since there are 10 shots available for each subject, a full rotating series takes 10 times.

Third in imposter rotation, role of imposter is rotated to another subject, e.g., 39th subject (M39). In other words, M39 becomes imposter and M40 becomes client. This imposer rotation only ends when every subject get a chance to act as imposter, thus a full imposter rotation make 40 rotations for 40 subjects.

A full rotating series happens in every imposter rotation. In other words, every time when there is a new combination of client and imposter, it will begin a full rotating series which is mentioned in second step. Throughout repeated series and imposters rotations in $\mathrm{AT}$ and $\mathrm{T}$ dataset, it finally yields a total amount of $40 \times 10 \times 39=15600$ scores for client and imposter verifications respectively. Each subject obtains one set of 390 authentic scores and one set of 390 imposter scores to plot client and imposter distribution.

PROTO1 is also applied in verification using CBE face database. Throughout all repetitions, it yielded a total amount of $40 \times 8 \times 39=12480$ scores in both client and imposter verifications. Each subject obtains one set of $8 \times 39=312$ authentic scores (same for imposter).

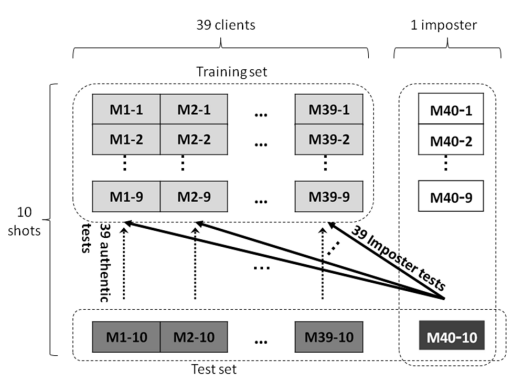

Fig. 11: PROTO1 protocol for $\mathrm{AT}$ and $\mathrm{T}$ dataset in allocation of training set, test set and imposter, where 39 authentic tests and 39 imposter tests are performed
PROTO1 employs class specific evaluations. In class specific evaluation, every client possesses own threshold and EER. In this experiment, all thresholds are set a posterior.

PROTO2 follows the testing protocol as in Wang et al. (2007). Five images per subject in AT and $\mathrm{T}$ face database are used as training samples and the rest 5 images per subject are set as testing samples. Figure 12 shows PROTO2 protocol where shot 1 of M1 in training set is under access by all testing samples. This shot 1 of M1 corresponds to 5 authentic tests and 195 imposter tests.

The whole verification involves all training samples. Every training sample is able to give 5 authentic scores and 195 imposter scores. It sums up a total of $5 \times 40=200$ authentic scores and $195 \times 40=7800$ imposter scores. On the other hand, PROTO2 is applied on CBE face database with 5 training images and 3 testing images per subject. It gives a total of $3 \times 40=120$ authentic scores and $117 \times 40=4680$ imposter scores.

PROTO2 employs global evaluations. In global evaluation, there is only one threshold for all clients, which is set a posterior in this experiment.

Verification parameter selection: For PROTO1, PC for PCA and LDA are selected based on study in face identification.

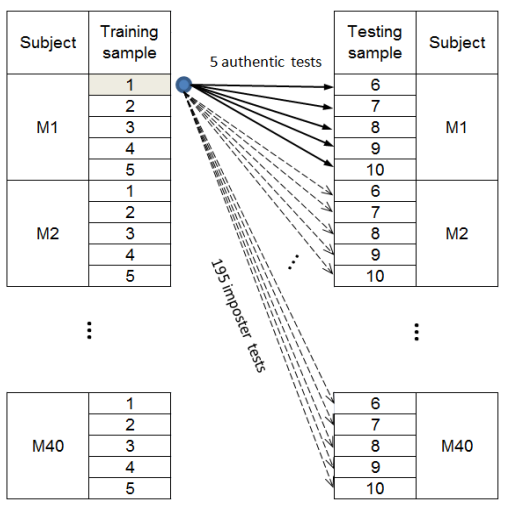

Fig. 12: Example of PROTO2 protocol

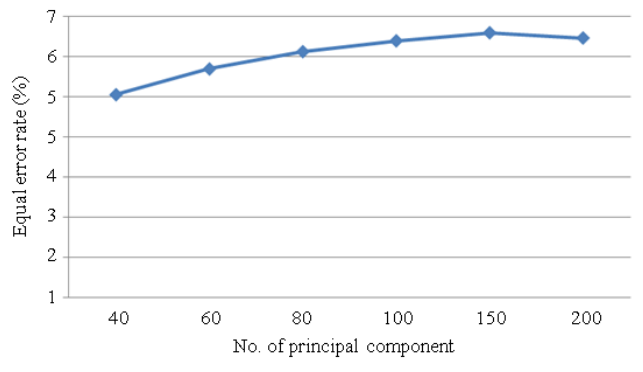

Fig. 13: EER performances of PCA under varying PC using $\mathrm{AT}$ and $\mathrm{T}$ database 
For PROTO2, PC is selected for PCA and LDA for AT and T dataset, where 200 authentic scores and 7800 imposter scores were collected. EER performance of PCA using different PC is shown in Fig. 13. EER performance is getting worse when PC increases. PCA obtains lowest EER at $\mathrm{PC}=40$.

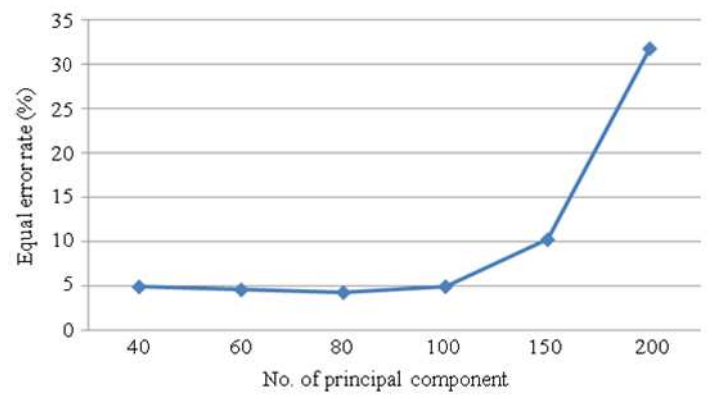

Fig. 14: EER performances of LDA under varying PC using $\mathrm{AT}$ and $\mathrm{T}$ database

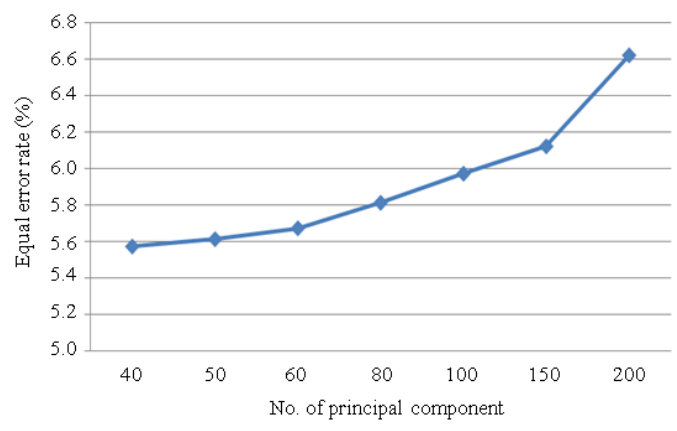

Fig. 15: EER performances of PCA under varying PC using CBE dataset

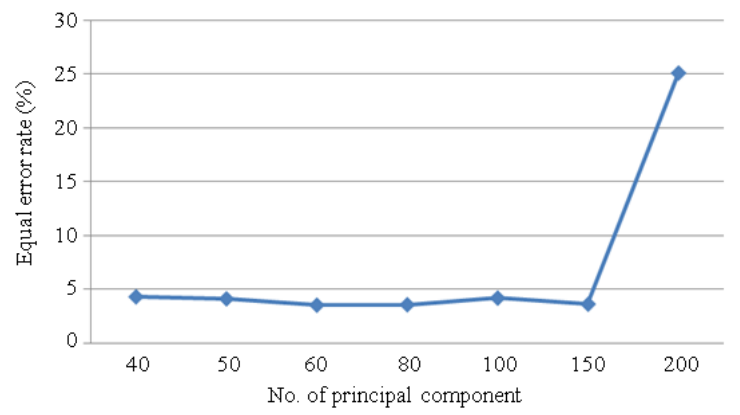

Fig. 16: EER performances of LDA under varying PC using CBE dataset

Table 1: Number of principal components proposed for $\mathrm{AT}$ and $\mathrm{T}$ and CBE database

\begin{tabular}{lllll}
\hline Database & \multicolumn{2}{l}{ Identification } & \multicolumn{2}{l}{ Verification (PROTO2) } \\
& ---------------- & ---------------- \\
& PCA & LDA & PCA & LDA \\
\hline AT and T & 40 & 40 & 40 & 80 \\
CBE & 60 & 45 & 40 & 60 \\
\hline
\end{tabular}

Effect of varied PC is investigated using LDA and the experimental results are shown in Fig. 14. LDA EER performance is around 5\% within $\mathrm{PC}=40$ and $\mathrm{PC}=100$. It begins deteriorating drastically when exceeding $P C=150$. Worst EER is obtained at $\mathrm{PC}=200$ while lowest EER is achieved at $\mathrm{PC}=80$.

The same investigation on varying $\mathrm{PC}$ was applied for CBE face dataset. Figure 15 and 16 show EER performances of PCA and LDA respectively. The graphs behave similarly as in AT and T database where better EER are obtained at lower PC. From the experiments, $\mathrm{PC}=40$ is selected for PCA while $\mathrm{PC}=60$ for LDA.

Proposed parameter: Table 1 shows number of principal components selected for $\mathrm{AT}$ and $\mathrm{T}$ and $\mathrm{CBE}$ database based on previous investigations. For verification PROTO1, the selection of PC is same as identification based on assumption that it is optimum.

\section{RESULTS}

Face identification using PCA and LDA were implemented based on parameters selected in previous section. For more reliable evaluations, different combinations of training set and testing set were used. Table 2 shows the identification performance of algorithms in six different ways of partitions using 40 subjects in the AT and $\mathrm{T}$ face database. The number 1-10 under the columns of training set and testing set represent 10 different images of a single subject (Fig. 5). For CBE Face Database, identification performances of algorithms using 4 different combinations of training and testing sets for 40 subjects are shown in Table 3. Improvement of LDA over PCA is $2.3 \%$ (AT and T) and $6.4 \%$ (CBE).

Table 2: Identification performances of algorithms for 40 subjects using 6 different ways for the AT and T face database

\begin{tabular}{lllll}
\hline & Training & Training & \multicolumn{2}{l}{ Recognition rate (\%) } \\
Set & set & set & PCA & LDA \\
\hline 1 & $1,2,3,4,5$ & $6,7,8,9,10$ & 88.50 & 91.00 \\
2 & $2,3,4,5,6$ & $1,7,8,9,10$ & 94.00 & 94.50 \\
3 & $3,4,5,6,7$ & $1,2,8,9,10$ & 95.00 & 97.00 \\
4 & $4,5,6,7,8$ & $1,2,3,9,10$ & 92.00 & 96.00 \\
5 & $5,6,7,8,9$ & $1,2,3,4,10$ & 92.50 & 94.00 \\
6 & $6,7,8,9,10$ & $1,2,3,4,5$ & 89.50 & 92.50 \\
Average & & & 91.90 & 94.20 \\
\hline
\end{tabular}

Table 3: Identification performances of algorithms for 40 subjects using 6 different ways for the CBE face database

\begin{tabular}{lllll}
\hline & Training & Testing & \multicolumn{2}{c}{ Recognition rate (\%) } \\
Set & set & set & PCA & LDA \\
\hline 1 & $1,2,3,4,5$ & $6,7,8$ & 73.3 & 77.5 \\
2 & $2,3,4,5,6$ & $1,7,8$ & 70.0 & 78.3 \\
3 & $3,4,5,6,7$ & $1,2,8$ & 76.7 & 85.0 \\
4 & $4,5,6,7,8$ & $1,2,3$ & 86.7 & 91.7 \\
Average & & & 76.7 & 83.1 \\
\hline
\end{tabular}


Table 4: Verification performances of algorithms for 40 subjects

\begin{tabular}{lllll}
\hline & \multicolumn{2}{l}{ EER (PROTO1) } & \multicolumn{2}{l}{ EER (PROTO2) (\%) } \\
Database & PCA & LDA & PCA & LDA \\
\hline AT and T & 1.15 & 0.78 & 5.05 & 4.20 \\
CBE & 7.30 & 5.81 & 5.57 & 3.53 \\
\hline
\end{tabular}

Verification performances of algorithms based on two protocols were conducted for 40 subjects using AT and $\mathrm{T}$ and $\mathrm{CBE}$ face database. Results are shown in Table 4. Compared with PCA, LDA is found achieving lower EER of $0.78 \%$ (PROTO1) and $5.81 \%$ (PROTO2) on AT and $\mathrm{T}$ dataset and $4.2 \%$ (PROTO1) and $3.53 \%$ (PROTO2) on CBE dataset.

\section{DISCUSSION}

Algorithms: First, PCA and LDA performances in face identification for the AT and $\mathrm{T}$ face database and $\mathrm{CBE}$ Face Database are reviewed. It is found that, for both database, LDA-based method successfully achieves better performances than PCA-based method. It proves that subspace LDA is able to provide more discriminative features to classifiers. It should be highlighted that, LDA improvement is especially significant for Euclidean distance in $\mathrm{CBE}$ face database.

Second, PCA and LDA performances in face verification are reviewed. For both PROTO1 and PROTO2, LDA-based method successfully achieves better performances than PCA-based method. It proves the feasibility of subspace LDA for face verification compared with PCA.

Database: The overall algorithms performances in CBE face database are not as good as AT and T. There are two main mains reasons: (1) better quality of the pictures in the AT and T face database, (2) higher difficulty of CBE Face Database due to variations in face details and head orientations.

\section{CONCLUSION}

This study has investigated the appearance based face recognition techniques in two aspects of face biometrics i.e., face identification and face verification. Appearance based techniques can solve the constraints of typical feature-based methods which heavily rely on face features detection and extraction. Among appearance based techniques, PCA and LDA are commonly used. It is worthy to study PCA and LDA as many advanced face recognition techniques have their foundations rooted on these techniques. From the experimental results, LDA is proven to have better performance than PCA in face biometrics.

\section{ACKNOWLEDGEMENT}

This research project is supported by Central of Biomedical Engineering (CBE) at University
Technology Malaysia and funded by Minister Of Science and Technology (MOSTI), Malaysia.

\section{REFERENCES}

Belhumeur, P.N., J.P. Hespanha and D.J. Kriegman, 1997. Eigenfaces Vs Fisherfaces: Recognition using class specific linear projection. IEEE Trans. Patt. Anal. Mach. Intell., 19: 711-720. http://www1.cs.columbia.edu/ belhumeur/journal/f isherface-pami97.pdf

Goudelis, G., S. Zafeiriou, A. Tefas and I. Pitas, 2007. Class-specific kernel-discriminant analysis for face verification. IEEE Trans. Inform. Forens. Security, 2: 570-587. DOI: 10.1109/TIFS.2007.902915

Kirby, M. and L. Sirovich, 1990. Application of the Karhunen-Loève procedure for the characterization of human faces. IEEE Trans. Patt. Anal. Mach. Intell., 12: 103-108. DOI: 10.1109/34.41390

Kotropoulos, C., A. Tefas and I. Pitas, 2000. Frontal face authentication using discriminating grids with morphological feature vectors. IEEE Trans. Multimedia, 2. pp: 14-26. DOI: $10.1109 / 6046.825791$

Martinez, A.M. and A.C. Kak, 2001. PCA versus LDA. IEEE Trans. Patt. Anal. Mach. Intell., 23: 228-233. DOI: $10.1109 / 34.908974$

Ruiz-del Solar, J. and P. Navarrete, 2005. Eigenspacebased face recognition: A comparative study of different approaches. IEEE Trans. Syst. Man Cybernet., 16: 817-830. http://www.cwr.cl/webfaces/articulos/his2002.pdf

Turk, M. and A. Pentland, 1991. Eigenfaces for recognition. J. Cognit. Neurosci., 3: 71-86. http://www.cs.cornell.edu/Courses/cs322/2007sp/sl ides/pca-figs.pdf

Wang, C.B., Y.P. Li, H.Z. Zhang and L. Wang, 2007. Classifier discriminant analysis for face verification based on FAR-score normalization. Proceeding of IEEE Symposium on Foundations of Computational Intelligence, Apr. 1-5, IEEE Xplore Press, Honolulu, pp: 476-480. DOI: 10.1109/FOCI.2007.371515

Zhao, W., R. Chellappa and A. Krishnaswamy, 1998. Discriminant analysis of principal components for face recognition. Proceedings of the 3rd International Conference on Automatic Face and Gesture Recognition, Apr. 14-16, IEEE Computer Society, USA., pp: 336-341. DOI: 10.1109/AFGR.1998.670971

Zhao, W., R. Chellapa, R.J. Phillips and A. Rosenfeld, 2003. Face recognition: A literature survey. ACM Comput. Surveys, 35: 399-458. DOI: $10.1145 / 954339.954342$ 